\title{
Orbital Resonances in the Solar Nebula: Strengths and Weaknesses
}

\author{
Renu Malhotra \\ Lunar and Planetary Institute, Houston. Texas 77058 \\ E-mail: renu(t $t$ lpis39.jsc.nasa.gov \\ Received March 31, 1993; revised June 10, 1993
}

\begin{abstract}
A planetesimal moving in the Solar Nebula experiences an aerodynamic drag which causes its orbit to circularize and shrink. However, resonant perturbations from a protoplanet interior to the planetesimal's orbit can counteract both the orbital decay and the damping of the eccentricity: the planetesimal can be captured into an orbital resonance and its eccentricity pumped up to a modestly high equilibrium value. Thus, orbital resonances constitute (partial) barriers to the delivery of planetesimals into the feeding zone of the protoplanet. We have established the characteristics of the phenomenon of resonance capture by gas drag in the circular restricted three-body approximation. We have determined the strengths of the equilibrium resonant orbits with respect to impulsive velocity perturbations. We conclude that planetesimals captured in orbital resonances are quite vulnerable to being dislocated from these orbits by mutual planetesimal interactions, but that the resonances are effective in slowing down the rate of orbital decay of planetesimals. Only very small bodies, $\leqslant 100 \mathrm{~m}$, are able to reach a $\sim 1 M_{\oplus}$ protoplanet without being slowed down by resonances. 1993 Academic Press, Inc
\end{abstract}

\section{INTRODUCTION}

The currently popular paradigm for the formation of the planets (see Wetherill (1989) for a recent review) starts with 1- to 10-km-diameter bodies- "planetesimals"-as the building blocks. These are presumably the result of an early agglomeration process from much smaller solid grains present in the Solar Nebula. Large numbers of planetesimals are thought to accumulate by means of a "runaway" process into a few planetary embryos of $\mathcal{O}(0.01-0.1) M_{\oplus}$ in the terrestrial planet region and $\mathcal{O}(1-5) M_{\oplus}$ at the present orbit of Jupiter. At the end of the runaway, these planetary embryos have depleted the supply of solid material in their "feeding" zones and are to be found in relatively isolated orbits. Further growth

Presented at the Planet Formation program held at the Institute for Theoretical Physics of the University of California at Santa Barbara, in Fall 1992. requires a means of delivering material to the feeding zone and/or direct merger of the runaway bodies.

Most of the recent calculations of the formation of planetary embryos have neglected the presence of nebular gas. In the early stages of the accumulation of the planets, solid bodies undoubtedly coexisted with the dominant gaseous component of the Solar Nebula. In particular, the accumulation of the cores of the jovian planets occurred before the dispersal of the nebular gas. Planetesimals moving through the gas would experience an aerodynamic drag that would damp their random motions (i.e., eccentricities and inclinations) and cause their orbits to spiral into smaller heliocentric distances (Adachi et al. 1976). Naively, then, one would expect that this inward flow of solid material would enhance the mass supply rate to the accretion zone of a planetary embryo embedded in the Solar Nebula. However, this does not necessarily occur since planetesimals would also be subject to mutual gravitational interactions. In particular, resonant gravitational perturbations from the protoplanet can counteract the effects of gas drag forces, leading to orbital resonance locks. A planetesimal's orbit would then cease to decay, and its major axis and eccentricity would attain equilibrium values. This was first pointed out in Weidenschilling and Davis (1985). The phenomenon is similar to the "mode-locking" phenomena that occur in many other natural systems of coupled oscillators.

The resonance trapping phenomenon acts as a barrier to the planetesimals' inward flow induced by the gas drag. However, it is only a partial barrier. In this paper, we describe the results of a systematic study of the permeability of this dynamical barrier. In order to elucidate the basic phenomenon, we consider the simplest case of the circular restricted three-body problem and use a very simple prescription for the gas drag force. We have carried out extensive numerical simulations to determine the orbital evolution of a planetesimal upon passage through a first-order orbital resonance with a protoplanet interior to its orbit. We describe the conditions for capture into 
. 
resonance and the characteristics of the equilibrium resonant orbit. The numerical results have been compared with analytical theory for adiabatic resonance passage (Henrard and Lemaitre 1983, Borderies and Goldreich 1984, Dermott et al. 1988, Malhotra 1988). We find that the theory gives reliable predictions for the evolution. In a more realistic scenario, planetesimals will suffer gravitational encounters and inelastic collisions with other planetesimals. The role and effectiveness of resonance capture in the presence of these processes needs to be examined. As a crude approximation, we simulate the effect of a gravitational encounter or collision by a velocity kick, $\delta \mathbf{v}$, imparted to the planetesimal. In this manner, we have determined the strength and weakness of a resonance with respect to planetesimal-planetesimal collisions. We demonstrate that in general, a planetesimal may be knocked out of an orbital resonance with a relatively small $\delta v$ imparted in the tangential direction, whereas the resonance is stable against much larger $\delta v$ 's imparted in the radial direction. We estimate that velocity kicks due to mutual planetesimal interactions are likely to exceed the strengths of resonances; as a result, the resonance traps are effective only in slowing down orbital decay, not completely halting it.

The paper is organized as follows. In Section II we describe the physical model we assumed. Section III summarizes relevant analytic estimates obtained from the adiabatic theory of resonance passage in the circular restricted three body problem. In Section IV we describe the results of numerical simulations and compare them with the analytic estimates. In Section V we discuss the strengths and weaknesses of orbital resonances with respect to planetesimal encounters. In Section VI we summarize our conclusions.

\section{THE PHYSICAL MODEL}

We assume that a planetesimal moves in the midplane of the Solar Nebula in a heliocentric orbit in the presence of nebular gas of constant density and is perturbed by a protoplanet which also moves in the midplane in an orbit interior to that of the planetesimal. We assume the restricted three-body formalism and neglect any evolution of the protoplanet's orbit. In heliocentric coordinates, the equations of motion for the planetesimal's position and velocity vectors are given by

$$
\begin{aligned}
& \dot{\mathbf{r}}=\mathbf{v}, \\
& \dot{\mathbf{v}}=-(1-\mu) \frac{\mathbf{r}}{r^{3}}-\mu\left(\frac{\mathbf{r}-\mathbf{r}_{\mathrm{p}}}{\left|\mathbf{r}-\mathbf{r}_{\mathrm{p}}\right|^{3}}-\frac{\mathbf{r}_{\mathrm{p}}}{r_{\mathrm{p}}^{3}}\right)+\mathbf{f}_{\mathrm{d}} .
\end{aligned}
$$

We choose units such that the gravitational constant $G=1$, and $M_{\odot}+M_{\mathrm{p}}=1$ (where $M_{\ominus}$ and $M_{\mathrm{p}}$ are the masses of the Sun and the protoplanet, respectively). The parameter $\mu$ is the mass of the protoplanet in units of the total mass $M_{\odot}+M_{\mathrm{p}}$,

$$
\mu=\frac{M_{\mathrm{p}}}{M_{0}+M_{\mathrm{p}}},
$$

$\mathbf{r}_{\mathrm{p}}$ is the heliocentric position vector of the protoplanet, and $\mathbf{f}_{\mathrm{d}}$ is the gas drag acceleration; in general it opposes the velocity of the planetesimal relative to the gas, but depends essentially upon the character of the relative motion (Landau and Lifshitz 1987, Adachi et al. 1976).

We will adopt, for specificity, the following numerical values of the physical parameters of the primordial Solar Nebula in the vicinity of Jupiter's orbit. (Although our considerations have wider applicability, we focus on these conditions at Jupiter's orbit because planetary embryo accumulation here undoubtedly occurred in the presence of nebular gas.) These values are used in the subsequent sections without further reference. The distance of the protoplanet from the Sun is assumed to be $a_{\mathrm{p}}=5 \mathrm{AU}=$ $7.5 \times 10^{13} \mathrm{~cm}$, and its circular orbital velocity is $v_{\text {kep }}=$ $1.3 \times 10^{6} \mathrm{~cm} \mathrm{sec}^{-1}$. An ambient nebular gas density $\rho_{\mathrm{gas}}=10^{-10} \mathrm{~g} \mathrm{~cm}^{-3}$ and temperature, $T=300 \mathrm{~K}$, are assumed. At this temperature, the mean thermal speed of gas molecules is $c=\left(3 k T / \pi m_{\mathrm{H}_{2}}\right)^{1 / 2} \approx 2 \times 10^{5} \mathrm{~cm} \mathrm{sec}^{-1}$. The molecular viscosity of the gas is then given by $\nu=$ $m_{\mathrm{H}_{2}} \mathrm{c} / 3 \sigma \approx 1 \times 10^{-4} \mathrm{~g} \mathrm{~cm}^{-1} \mathrm{sec}^{-1}$ (where we have used $\sigma=2 \times 10^{-15} \mathrm{~cm}^{2}$ for the collision cross section of molecular hydrogen). The gas is assumed to be in nearKeplerian rotation. The pressure support in the gas causes the gas velocity to be slightly smaller than the local circular Keplerian velocity: $1-\left(v_{\text {gas }} / v_{\text {kep }}\right) \approx(1 / 2)\left(c / v_{\text {kep }}\right)^{2} \approx$ $5 \times 10^{-3}$. The bulk density of the planetesimals will be taken to be $\rho=2 \mathrm{~g} \mathrm{~cm}^{-3}$.

Taking the relative velocity, $u$, of a planetesimal with respect to the gas to be about $1 \%$ of the Keplerian velocity, we have $u \approx 1.3 \times 10^{4} \mathrm{~cm} / \mathrm{sec}$. (Note that this relative motion is subsonic, but just barely so!) For the relative motion of a planetesimal of radius $s$ through the gas, the Reynolds number, $\operatorname{Re}=2 s \rho_{\text {gas }} u / \nu$, has a simple mnemonic form,

$$
\operatorname{Re} \approx \frac{s}{1 \mathrm{~m}} .
$$

Therefore, for planetesimals having radii greatly exceeding $10 \mathrm{~m}, \operatorname{Re} \gg 10$. At high values of $R e$, it is an experimentally observed fact that the drag acceleration is independent of the viscosity and is well represented by (Landau and Lifshitz 1987)

$$
\mathbf{f}_{\mathrm{d}}=-K u \mathbf{u} ; \quad \mathbf{u}=\mathbf{v}-\mathbf{v}_{\text {gas }},
$$

where the coefficient $K$ is given by 


$$
K=\frac{C_{\mathrm{d}} \pi s^{2}}{2 m} \rho_{\mathrm{gas}}=\frac{3 C_{\mathrm{d}} \rho_{\mathrm{gas}}}{8 \rho s}
$$

where $m$ is the mass of the planetesimal, and $C_{\mathrm{d}}$ is a nondimensional coefficient whose magnitude is known from laboratory experiments to be nearly constant at a value of about 0.5 for $R e$ in the range $500-10^{5}$. Thus, for example, the numerical value of $K$ is $0.014 / \mathrm{AU}$ for a planetesimal $100 \mathrm{~m}$ in radius. We note that for gas densities smaller than assumed above, or smaller sizes of planetesimal, or smaller relative velocities, the Reynolds number could well drop below the range of applicability of Eq. (4). If $\mathrm{Re} \leqslant 10$, then Stokes' law would provide a better description. In this paper we confine our attention to a drag force in the form of Eq. (4).

In Eq.(4), $u$ is the velocity of the planetesimal relative to the gas velocity, $\mathbf{v}_{\mathrm{gas}}$, which we assume is given by

$$
\mathbf{v}_{\mathrm{gas}}(\mathbf{r})=(1-\eta) \mathbf{v}_{\mathrm{kep}}(\mathbf{r}),
$$

where $\eta \approx 0.005$ measures the slight offset (due to the partial pressure support) of the gas velocity with respect to the local circular keplerian velocity.

The equations of motion (Eqs. (1)) were integrated using a simple modification of a mixed variable symplectic scheme (Malhotra 1993), using at least 20 steps per orbit of the planetesimal. This numerical method is about an order of magnitude faster than conventional integrators such as the Bulirsh-Stoer integrator (Press et al. 1986). The reliability of the results was confirmed in a large number of cases by repeating the integrations using the Bulirsh-Stoer integrator.

\section{ANALYTICAL ESTIMATES}

The evolution of a keplerian orbit due to gas drag has been analyzed in Adachi et al. (1976). To lowest order in the small quantities $e$ and $\eta$, the variation of the orbital semimajor axis and eccentricity under the drag law of Eq. (4) are given by

$$
\begin{aligned}
& (\dot{a})_{\mathrm{drag}} \simeq-\frac{2}{\tau_{0}}(0.97 e+\eta) \eta a, \\
& (\dot{e})_{\mathrm{drag}} \simeq-\frac{1}{\tau_{0}}(0.77 e+\eta) e,
\end{aligned}
$$

where

$$
\tau_{0}=\frac{1}{K v_{\text {kep }}(a)}
$$

For relative velocity $u$ on the order of $1 \%$ of the local keplerian velocity, the orbital decay time scale is $\tau_{\mathrm{a}} \approx$
$2 \times 10^{6}(\mathrm{~s} / 1 \mathrm{~km})$ years, and the orbit circularization time scale is $\tau_{e} \approx 2 \times 10^{4}(s / 1 \mathrm{~km})$ years.

In the context of the circular restricted three-body problem, in the vicinity of a $j+1: j$ exterior resonance with the protoplanet, the critical resonance angle is defined by

$$
\phi=(j+1) \lambda-j \lambda_{p}-\varpi,
$$

where $\lambda$ and $\lambda_{p}$ are the mean longitudes of the planetesimal and protoplanet, respectively, and $\boldsymbol{\sigma}$ is the planetesimal's longitude of perihelion. The resonant gravitational torque from the protoplanet induces a variation of $a$ and $e$ given by

$$
\begin{aligned}
& (\dot{a})_{\text {res }}=-2(j+1) \mu n a e f(\alpha, j) \sin \phi, \\
& (\dot{e})_{\text {res }}=-\mu n f(\alpha, j) \sin \phi,
\end{aligned}
$$

where $n=a^{-3 / 2}$ is the mean motion of the planetesimal; $\alpha=1 / a$, and $f(\alpha, j)$ is a coefficient of the resonant term in the expansion of the protoplanet's gravitational potential, given in terms of Laplace coefficients, $b_{s}^{(j)}(\alpha)$, by (Brouwer and Clemence 1961)

$$
f(\alpha, j)=\frac{1}{2}\left(2 j+1+\alpha \frac{d}{d \alpha}\right) b_{1 / 2}^{(j)}(\alpha) .
$$

At exact resonance, $\alpha=\alpha_{\text {res }}=(j /(j+1))^{2 / 3}$, we have the asymptotic expression,

$$
f\left(\alpha_{\text {res }}, j\right) \simeq 0.80 j, \quad \text { for } j \gg 1 .
$$

For small rates of orbit evolution due to drag (Eqs. (7)), we can draw upon the adiabatic theory of resonance passage to determine the conditions for resonance capture. A review of this analysis is given in Peale (1986). In this theory, the three-body Hamiltonian is averaged to obtain a pendulum-like resonance Hamiltonian with one degree of freedom (corresponding to the resonance angle, $\phi)$. The drag-induced evolution is modeled as a drift of the particle across the slowly varying phase space trajectories determined by the resonance Hamiltonian. As the particle energy approaches that of the exact resonant orbit, a separatrix appears in the phase space which defines the resonance and nonresonant regions. Depending upon the initial orbit of the particle and the rate of drag-induced drift, the particle may be captured into the resonance region or may continue to evolve past the resonant orbit.'

\footnotetext{
${ }^{1}$ We note that this theory neglects the damping of the orbital eccentricity by the drag force. This is a shortcoming that is not of much import for the approach to the resonance, but it makes the theory inadequate for the evolution subsequent to resonance capture when the damping rate becomes comparable to the rate of resonant excitation of the eccentricity.
} 
Qualitatively, the conditions for resonance capture require that (i) the planetesimal eccentricity upon approach to the resonance be not too high, and (ii) the rate of decay of the orbit be sufficiently slow. We quantify these conditions below.

For the first condition, the critical eccentricity is given by (cf. Henrard and Lemaitre 1983, Borderies and Goldreich 1984, Dermott et al. 1988):

$$
e_{\mathrm{crit}}=\left[\frac{2 \sqrt{6} \mu f\left(\alpha_{\mathrm{res}}, j\right)}{(j+1)^{2}}\right]^{1 / 3} \simeq 1.58\left[\frac{j \mu}{(j+1)^{2}}\right]^{1 / 3} .
$$

If the second ("adiabatic") condition is satisfied (and we quantify that below), then capture into the resonance is certain for $e \leq e_{\text {crit. }}$. For higher eccentricities, the capture probability drops off rapidly:

$$
P_{\text {capture }} \simeq \frac{2}{3^{3 / 4} \pi}\left(\frac{e_{\text {crit }}}{e}\right)^{3 / 2},
$$

where $e$ is the initial eccentricity long before the approach to the resonance. If the adiabatic condition is only weakly satisfied, then capture into resonance can still occur, but with probability smaller than that corresponding to the adiabatic case (Malhotra and Dermott 1990). Since planetesimal eccentricities are damped efficiently by nebular gas drag, the condition $e<e_{\text {crit }}$ is likely to be satisfied in many cases. Then, only those planetesimals that can overcome the adiabatic threshold may pass through the resonance barrier.

The second condition for resonance capture may be quantified by requiring that the change in the semimajor axis due to gas drag in a characteristic resonance libration period, $T_{t}$, be smaller than the amplitude $(\Delta a)_{t}$ of resonant perturbations of the semimajor axis (Dermott et al. 1988):

$$
(\dot{a})_{\mathrm{drag}} T_{\ell} \ll(\Delta a)_{\ell} .
$$

$T_{f^{\prime}}$ and $(\Delta a)_{\ell}$ are given by

$$
\left(\frac{\Delta a}{a}\right)_{t}=\frac{8 \pi\left(n T_{f}\right)^{-1}}{3(j+1)}=4\left[\frac{1}{3} \mu f\left(\alpha_{\mathrm{res}}, j\right) e\right]^{1 / 2} .
$$

From Eqs. (7), (15), and (16), the adiabatic condition is found to be:

$$
a K \eta\left(0.97+\frac{\eta}{e}\right) \ll 0.25 j(j+1) \mu .
$$

We can define a threshold mass,

$$
\mu_{\mathrm{th}}=\frac{4.0}{C_{\mathrm{ad}}^{\prime}} \frac{a K \eta(0.97+\eta / e)}{j(j+1)},
$$

such that for a given planetesimal (hence $K$ ), a protoplanet of mass $\mu>\mu_{\text {th }}$ is required to halt the orbital decay of the planetesimal by capture in the $j+1: j$ resonance. $C_{\text {ad }}^{\prime}$ is a numerical constant that measures how well the inequality (15) needs to be satisfied for resonance capture to occur (i.e., $C_{\text {ad }}^{\prime}$ is the ratio of the left-hand side to the right-hand side). We call it the adiabatic parameter.

We note that $\mu_{\mathrm{th}}$ defined above depends upon the planetesimal's orbital eccentricity as it approaches the resonance. As mentioned previously, if the initial eccentricity is too large, resonance capture is unlikely to occur, and no other conditions are relevant. However, orbit circularization by gas drag is quite rapid; therefore, we must consider the case of low-eccentricity orbits. If the initial eccentricity far away from the resonance is very small, then upon approach to the resonance, there is an increasing eccentricity forced upon the planetesimal. An appropriate value of $e$ to use in the expression for $\mu_{\mathrm{th}}$ above is the magnitude, $e^{*}$, of the forced eccentricity when the resonance separatrix first appears in the phase space. This value is (cf. Malhotra 1988)

$$
e^{*}=(2 / 3)^{1 / 2} e_{\text {cril }},
$$

where $e_{\text {crit }}$ was given in Eq. (13) above. For the parameter ranges of interest, we find that the magnitude of $e^{*}$ is likely to be comparable to $\eta$. Therefore, we will assume $\left(0.97+\eta / e^{*}\right) \approx$ constant, and absorb its value in the numerical factor, $C_{\mathrm{ad}}=C_{\mathrm{ad}}^{\prime} /(1+\eta / e)$; thus, we arrive at the following expression for $\mu_{\mathrm{th}}$ :

$$
\mu_{\mathrm{th}}=\frac{4.0}{C_{\mathrm{ad}}} \frac{a K \eta}{j(j+1)} .
$$

We show below that $C_{\mathrm{ad}}$ is insensitive to the system parameters, and that its numerical value is $\leqslant 3.3$.

In the evolution subsequent to capture into resonance, the planetesimal settles into an equilibrium orbit. This is easily seen by considering the total time rates of change of $a$ and $e$ :

$$
\begin{aligned}
& \dot{a}=-2(j+1) \mu n a e f(\alpha, j) \sin \phi-\frac{2}{\tau_{0}}(0.97 e+\eta) \eta a, \\
& \dot{e}=-\mu n f(\alpha, j) \sin \phi-\frac{1}{\tau_{0}}(0.77 e+\eta) e .
\end{aligned}
$$

The equilibrium resonant orbit is given by setting $\dot{a}$ and $\dot{e}$ to zero. This condition yields 


$$
\begin{aligned}
e_{\mathrm{eq}} & \simeq 1.12 \sqrt{\frac{\eta}{j+1}}, \\
\sin \phi_{\mathrm{eq}} & \simeq-1.21 \frac{\eta a K}{j(j+1) \mu},
\end{aligned}
$$

where corrections of $\mathcal{O}\left(\eta / e_{\mathrm{eq}}\right)$ have been neglected. The equilibrium eccentricity is independent of the drag parameter, $K$, and hence of the planetesimal size. For vanishingly small drag rates, $K \rightarrow 0$, we recover the classical result that the fixed points for a first-order resonance are at $\phi=0$ and $\phi=\pi$; a linear stability analysis shows that the latter is the center of libration in the resonance.

We can estimate the time scale to establish the equilibrium state as follows. Immediately upon capture into resonance, we have $\langle\dot{a}\rangle=0$, where \langle\rangle denote a time average over a characteristic resonance libration period. Therefore, from Eqs. (21),

$$
\langle\sin \phi\rangle=-\frac{1}{\tau_{0}} \frac{(0.97 e+\eta) \eta}{(j+1) \mu n e f(\alpha, j)}
$$

Inserting this into the equation for $\dot{e}$, we find that the secular variation of the eccentricity upon resonance capture is given by

$$
\left\langle\frac{\dot{e}}{e}\right\rangle=\frac{1}{\tau_{0}}\left[\frac{(0.97 e+\eta) \eta}{(j+1) e^{2}}-(0.77 e+\eta)\right] .
$$

The two terms on the right-hand side represent the resonant excitation and the gas-drag-induced damping, respectively. An explicit expression for $\langle e\rangle$ as a function of time is not easy to obtain from this deceptively simple equation. However, the time scale for the resonant excitation of the eccentricity is given by the inverse of the first term. By setting $e=e_{\mathrm{eq}}$ in that expression, we estimate the time to establish the equilibrium state to be

$$
\begin{aligned}
& \tau_{\mathrm{eq}} \sim\left(\tau_{0} / e_{\mathrm{eq}}\right) \\
& \quad \sim 3 \times 10^{2}(j+1)^{1 / 2}\left(\frac{s}{100 \mathrm{~m}}\right)\left(\frac{a_{\mathrm{p}}}{5 \mathrm{AU}}\right)^{1 / 2} \text { years. }
\end{aligned}
$$

$\tau_{\text {eq }}$ is very short compared to the orbital decay time scales.

A useful exercise at this point is to combine Eq. (20) with the expression for $\sin \phi_{\mathrm{eq}}$ (Eq. (22)) to obtain the equilibrium value of $\phi$ at the resonance capture threshold:

$$
\sin \phi_{\text {eq.th }} \simeq-0.30 C_{\text {ad }} .
$$

Now, because $|\sin \phi| \leq 1$, it follows that $C_{\mathrm{ad}} \leq 3.3$. It is worth pointing out that the insensitivity of the adiabatic parameter to the system parameters (planet mass, orbital elements of the particle, and the parameters of the gas) holds only for the first-order resonances that we consider here and is a consequence of the form of the drag law.

The estimates obtained above for the equilibrium resonant orbit (Eqs. (22)) are equivalent to those found previously by Weidenschilling and Davis (1985). (Beware, however, of typos in their paper). Those authors argued that the condition $\left|\sin \phi_{\text {eq }}\right| \leq 1$ defines a maximum value, $K_{\text {max }}$, for the stability of the equilibrium resonant orbit and determines the condition for resonance capture. In the notation used here, we get

$$
a K_{\max } \simeq \frac{(j+1) \mu}{\eta} f\left(\alpha_{\mathrm{res}}, j\right)
$$

This expression is similar to the adiabatic threshold determined by our Eq. (20) above. ${ }^{2}$

In the next section we compare the analytic estimates made above with the results of numerical calculations. We note here that this analysis is valid only for resonances sufficiently well separated that a single resonant term in the protoplanet's gravitational potential dominates the perturbations felt by the planetesimal. Sufficiently close to the protoplanet, first-order orbital resonances will not be well separated, and the perturbations will be a superposition of many resonances simultaneously. The response of a particle to overlapping resonances is chaotic (Chirikov 1979). The value, $j_{\mathrm{c}}$, such that all $j+1: j$ resonances with $j>j_{\mathrm{c}}$ in the vicinity of the protoplanet are overlapping is given by (Wisdom 1980)

$$
j_{\mathrm{c}}=0.5 \mu^{-2 / 7} .
$$

Therefore, we may with some justification consider the feeding zone of the protoplanet to extend to the $j_{\mathrm{c}}+1$ : $j_{\mathcal{c}}$ resonances interior and exterior to its orbit, i.e., a halfwidth

$$
\frac{\delta a}{a} \approx 1.5 \mu^{2 / 7}
$$

For $\mu<4 \times 10^{-5}$, the feeding zone is larger than the $\sim 3.5$ Hill radii that is often assumed.

\footnotetext{
2 We should point out here a technical difference between our calculations and those of Weidenschilling and Davis (1985) in arriving at the above condition for resonance capture. These authors implicitly assumed $e-C(\eta)^{1 / 2} \gg \eta$; this is a reasonable approximation for the equilibrium resonant orbit. However, whether capture occurs is determined by conditions at the approach to the resonance. We have argued above that, at the approach to the resonance, $e$ is comparable to $\eta$. Since the factor $\eta / e$ appears only as $(0.97+\eta / e)$ in the adiabatic condition, our results are not different, but they go to the heart of the mechanism of resonance capture.
} 

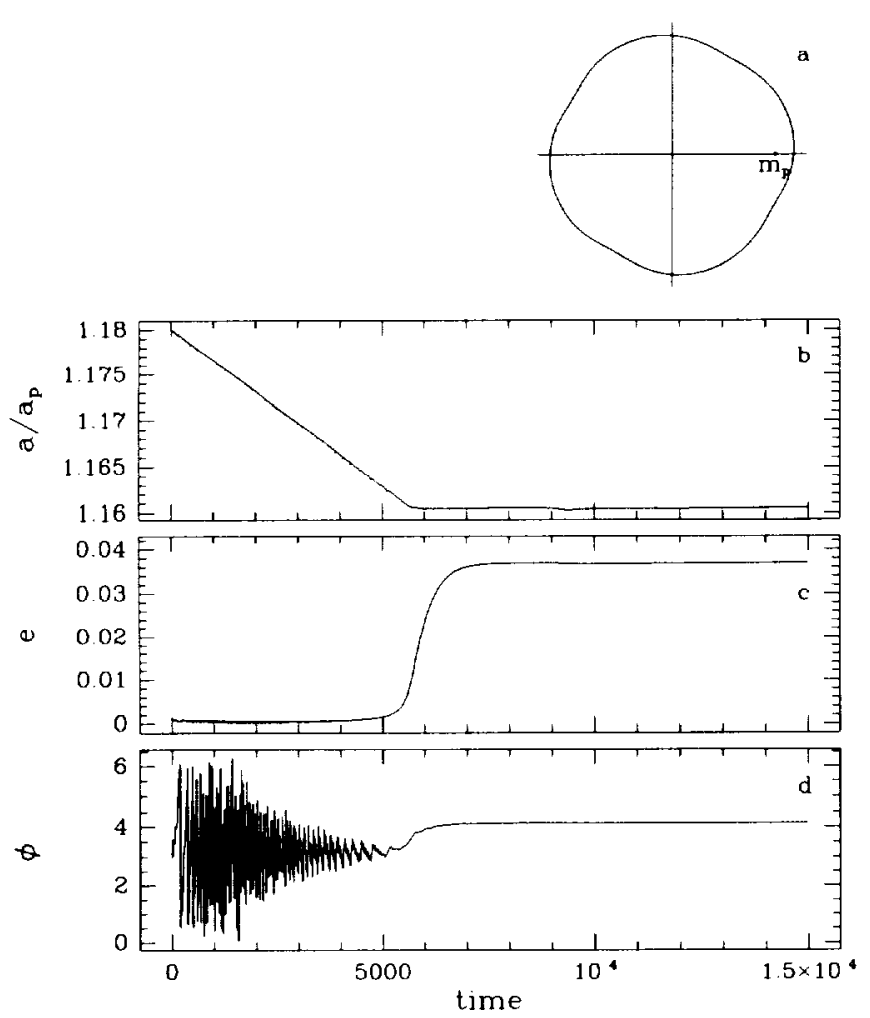

FIG. 1. An example of capture in $5: 4$ exterior resonance. (a) The equilibrium resonant orbit in the frame rotating with the Sun and the perturbing planet; note the characteristic fourfold symmetry $(j$-fold for a $j+1: j$ resonance). (b)-(d) The time evolution of the semimajor axis, eccentricity, and resonance angle; the unit of time is the orbital period of the perturbing planet. The planet mass and drag parameter in this example were $\mu=3 \times 10^{\circ}$ and $K=0.01$, respectively.

\section{NUMERICAL RESULTS}

In Fig. 1 we show the typical orbital evolution of a planetesimal upon capture in a first-order resonance with a protoplanet which moves on a circular orbit. Initially the semimajor axis decays and the eccentricity is damped. After the particle is captured in the resonance, the semimajor axis stabilizes at the resonant value, $a \simeq((j+1) /$ $j)^{2 / 3}$; the eccentricity and resonance angle approach their equilibrium values quite rapidly. We note that long before the equilibrium state is attained, the resonance angle may be found to be in libration about $\pi$. This libration is dynamically inconsequential-it arises merely due to the high rate of orbital precession induced by a small eccentricity in the neighborhood of a first-order resonance, and there is no net transfer of angular momentum from the protoplanet to the planetesimal during this stage. This is confirmed by the observation that the planetesimal eccentricity does not get excited and the semimajor axis continues its decay unaltered during this stage.
Figure 2 shows the evolution of a planetesimal's eccentricity upon passage through a $5: 4$ orbital resonance for several different protoplanet masses. It may be seen that for cases where $\mu$ is greater than a threshold value, $\mu_{1 \mathrm{~h}}$, the eccentricity evolution is independent of the value of $\mu$; the planetesimal is captured in the resonance and settles into an equilibrium state. For values of $\mu$ smaller than $\mu_{\mathrm{th}}$, resonance capture does not occur; the eccentricity is briefly excited to a modest value but is eventually damped away. The magnitude of this excitation decreases rapidly with the mass of the protoplanet.

In Figures 3, 4, and 5 we have summarized the results of an extensive series of numerical simulations of the resonance capture phenomenon. We simulated the evolution for two different values of the drag parameter: $a_{\mathrm{p}} \mathrm{K}=0.001$ and $a_{\mathrm{p}} K=0.01$, and a wide range of protoplanet mass, $\mu$. For reference, these two values of $K$ correspond to approximately 7.5 - and $0.75-\mathrm{km}$-radius planetesimals, respectively.

- Figure 3 shows the minimum protoplanet mass necessary for a planetesimal with a small initial eccentricity $\left(e<e_{\text {crii }}\right)$ to be captured into resonance; the dashed line indicates the adiabatic threshold defined by Eq. (20).

- Figure 4 shows the equilibrium eccentricity as a function of the integer, $j$; the numerical experiments confirm its insensitivity to the protoplanet mass and the planetesimal size.

- Figure 5 shows the numerically determined equilibrium value of the resonance angle at the capture threshold. There appears to be a weak dependence on the particle size and the integer $j$, such that the center of libration,

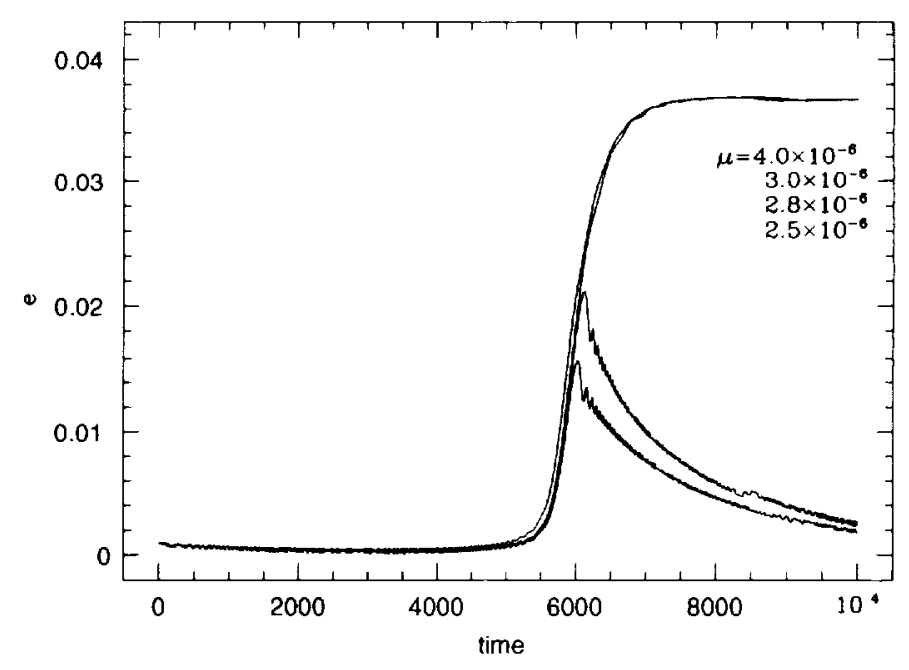

FIG. 2. The time evolution of a planetesimal's eccentricity upon passage through a $5: 4$ exterior resonance. Four cases, each corresponding to a different perturbing planet mass, $\mu$, are shown. For $\mu$ less than a certain threshold value, the planetesimal passes through the resonance without being captured. 


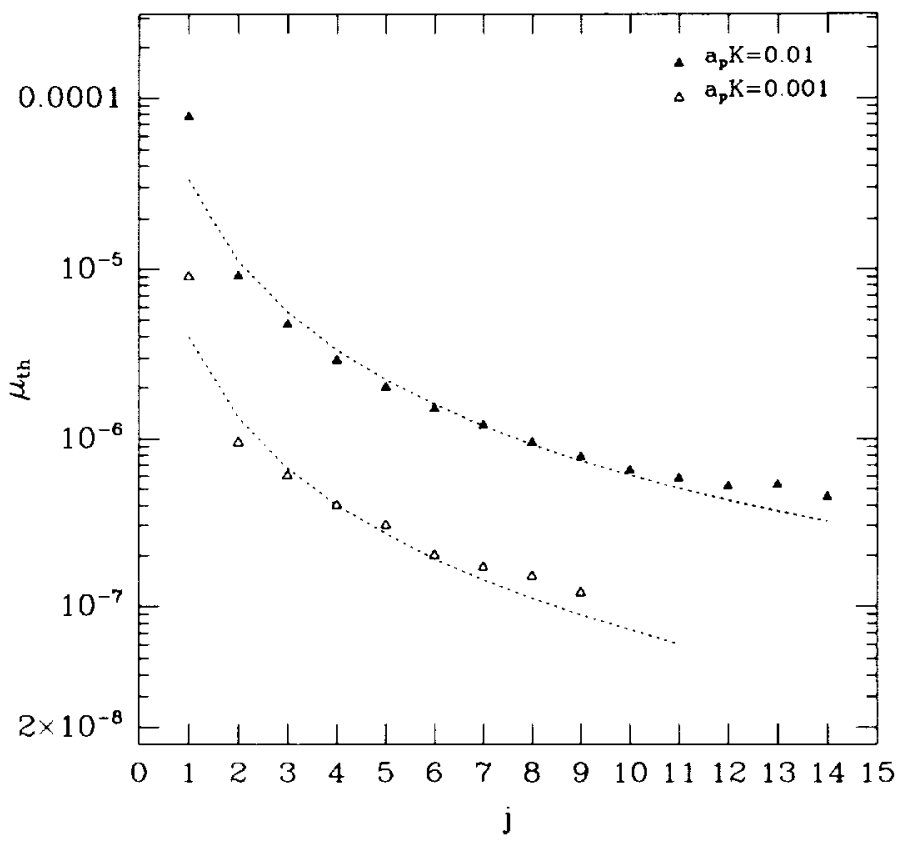

FIG. 3. The threshold planet mass for capture into $j+1: j$ exterior resonance. The points are from numerical experiments, the dashed line represents the analytical estimate of Eq. (20) with numerical coefficient $C_{\mathrm{ad}}=3.0$ for the case $K=0.01$, and $C_{\mathrm{ad}}=2.5$ for the case $K=0.001$.

$\phi_{\text {eq }}$, for smaller planetesimals at distant resonances is removed farther from the "classical" value of $\pi$.

These results show that in the restricted three-body formalism, the analytical estimates describe the dynamical evolution quite well.

However, for parameters close to the resonance capture threshold, the dynamics is very complicated and the analysis of Section III can be relied upon only broadly, but not in the utmost details. In particular, we find that the analytical estimate of $e_{\mathrm{eq}}$ is quite robust but $\sin \phi_{\mathrm{eq}}$ is not quite so. For example, Fig. 5 shows that at the capture threshold, the equilibrium values of the resonance angle hover close to $3 \pi / 2$; however, the resolution of our numerical experiments was too poor to make a meaningful interpretation of the deviations from $3 \pi / 2$. Recent work by Kary et al.(1993) discusses this issue in greater detail.

\section{STRENGTHS OF RESONANCES}

In this section, we consider the strength of a $j+1$ : $j$ exterior resonance against close encounters between planetesimals. As a crude approximation, we can model the effect of a planetesimal-planetesimal encounter by an instantaneous kick in velocity, $\delta \mathbf{v}$, imparted to each body which, in effect, reinitializes the orbital elements. As long as the new orbital elements remain within the resonance width, the planetesimal will remain trapped in the reso-

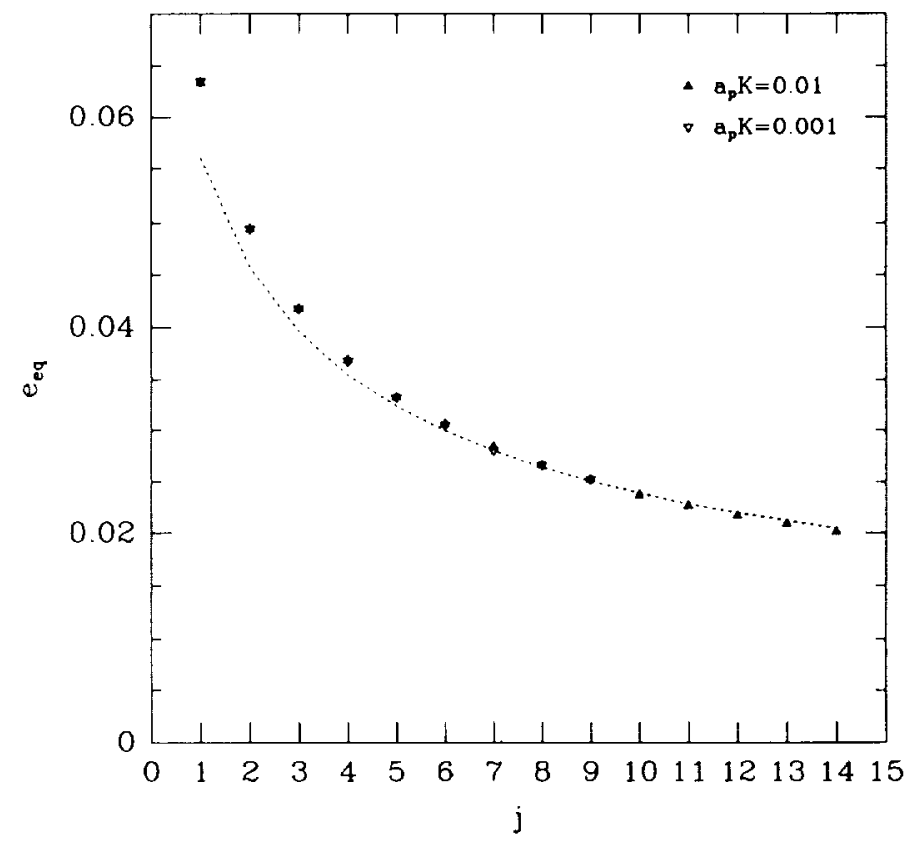

FIG. 4. The equilibrium eccentricity in $j+1: j$ exterior resonances, as a function of the integer, $j$. The points are from numerical experiments, the dashed line represents the analytical estimate of Eq. (22). $e_{\text {eq }}$ is insensitive to the planetesimal size and to the mass of the protoplanet.

nance. The resonance width varies sensitively with orientation in phase space. Therefore, we expect the strength of the resonance to be sensitive to the direction of the velocity change, $\delta \mathbf{v}$.

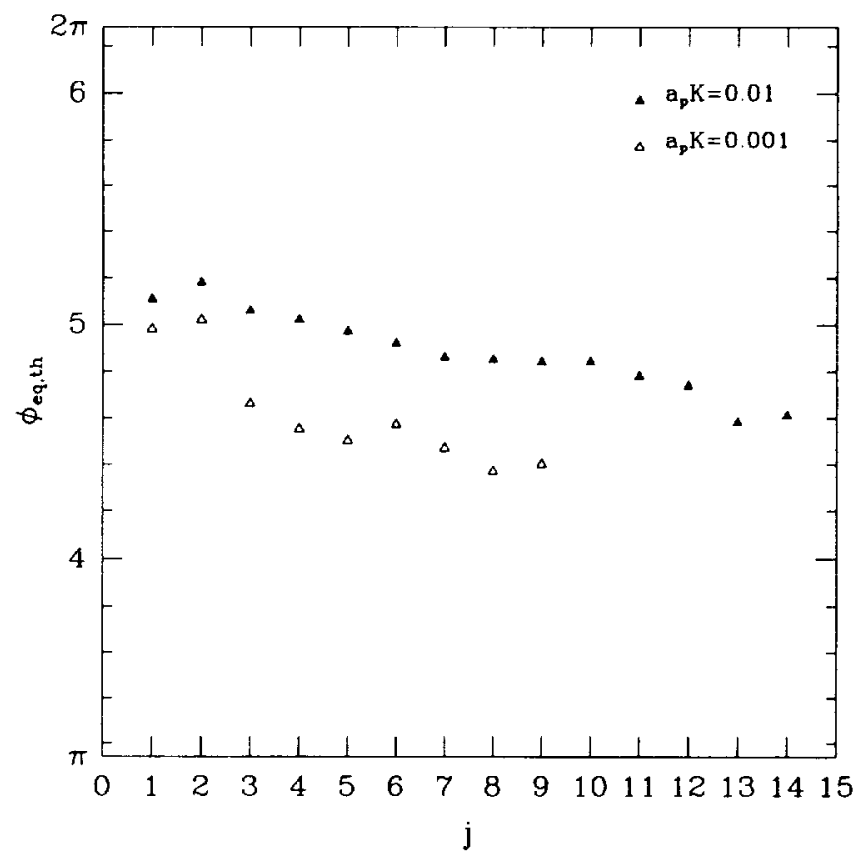

FIG. 5. The numerically determined equilibrium value of the resonance angle at the resonance capture threshold. 


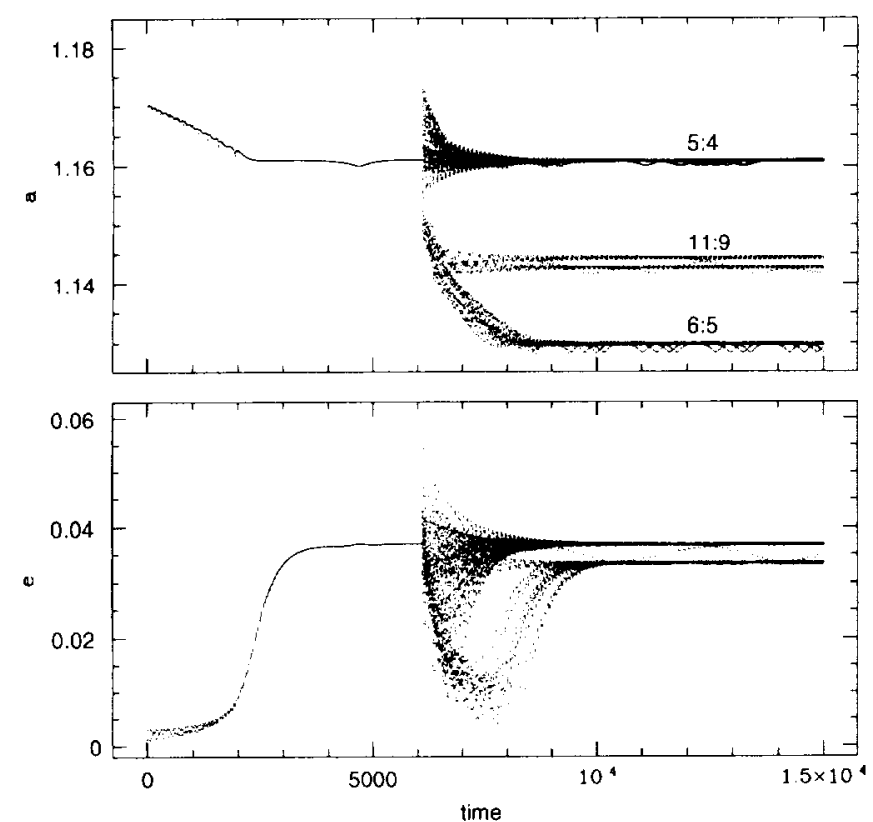

FIG. 6a. Time evolution of the semimajor axis and eccentricity of a set of 20 planetesimals $\left(K=0.01, \mu=3 \times 10^{-5}\right.$. (Unit of time is the orbital period of the planet.) All the planetesimals are at first captured in the $5: 4$ resonance. At time $=6103.7$, each planetesimal suffers a velocity kick of magnitude $\delta v=0.005$ but different orientation. In the subsequent evolution, some remain the $5: 4$ resonance, others are kicked out of the $5: 4$ but get captured in other resonances closer to the protoplanet.

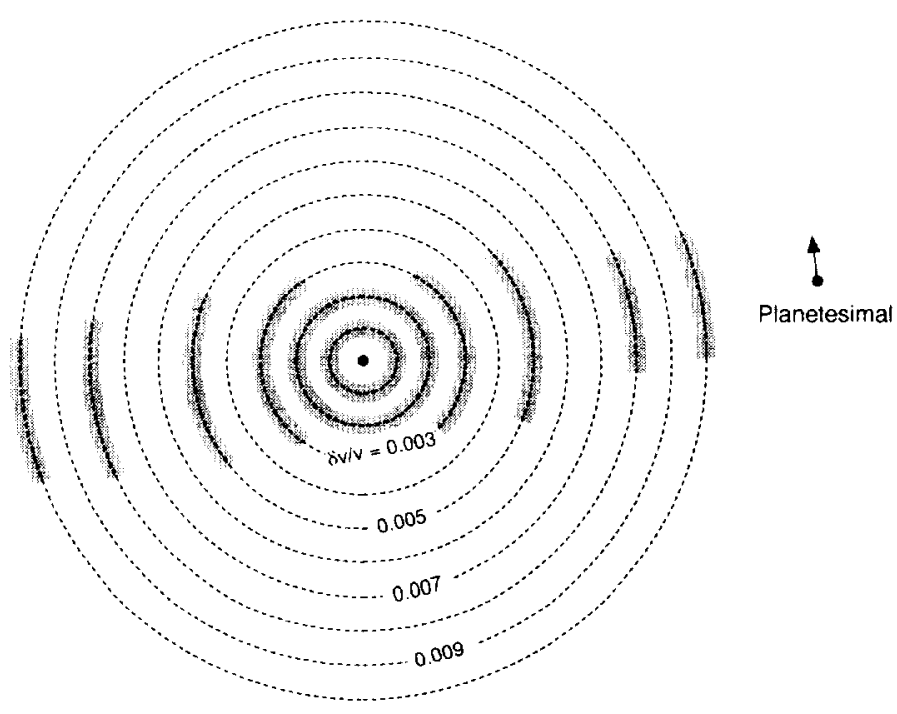

FIG. 6b. The strength of the $5: 4$ resonance with a $10 M_{\oplus}$ protoplanet. Shown is a summary of the results from numerical experiments of the type indicated in Fig. 6a: the shaded regions indicate the magnitude and orientation of an impulsive velocity kick that does not result in the planetesimal being ejected out of the resonance. The position of the particle at the time of the velocity kick is indicated by 1 . It is apparent that the resonance is far stronger against radial $\delta v$ 's than against tangential $\delta$ v's
We can estimate as follows the maximum $\delta v$ that a planetesimal captured in a resonance can suffer without being knocked out of the resonance. A $\delta v$ which is along the velocity vector of the planetesimal will produce a change in the semimajor axis, $\delta a \approx 2(\delta v / v) a$. The maximum half-width of the resonance along $a$ is simply $(\Delta a)_{t}$ (Eq. (16)) evaluated for the equilibrium resonant orbit (i.e., at $e=e_{\text {eq }}$, Eq. (22)). Thus, we obtain the following estimate:

$$
\left|\frac{\delta v}{v}\right|_{\mathrm{res}} \approx 1.1\left(\frac{j^{2}}{j+1}\right)^{1 / 4} \mu^{1 / 2} \eta^{1 / 4} \approx 0.29 j^{1 / 4} \mu^{1 / 2} .
$$

We have verified this estimate by means of numerical experiments. An example is shown in Figs. 6a and 6b. Figure $6 \mathrm{a}$ shows the evolution of a set of 20 planetesimals (with the same initial conditions) into a $5: 4$ exterior resonance with a $10 M_{\oplus}$ protoplanet. At a specified time into the evolution, each particle was imparted a velocity kick of magnitude $\delta v=5 \times 10^{-3}$ (but different orientation). It may be seen that some fraction of the particles remain in the resonance, or are knocked into higher orbits which are then recaptured into the $5: 4$ resonance; the rest are knocked out of the resonance and their orbits decay inward. (In the example shown, all of these eventually get captured into other resonances interior to the $5: 4$ resonance.) Figure $6 \mathrm{~b}$ summarizes the results of many such numerical experiments. It shows the strength of the resonance in terms of the magnitude and orientation of the velocity kicks necessary to disrupt the resonance: there is clearly a high degree of anisotropy. The theoretical estimate of Eq. (30) yields $\delta v / v=0.002$ for the width along the tangential direction; this is in very good agreement with the results shown in Fig. $6 \mathrm{~b}$.

We have performed similar experiments for several different resonances (and with differing masses of the protoplanet) and have found that Eq. (30) provides an excellent estimate of the resonance strength with respect to velocity kicks along a tangent to the planetesimal's orbit. Along the radial direction, the resonance strength is greater by factors of $\sim 4$ or more.

\section{CONCLUSIONS}

The conditions for resonance capture imply a high efficiency of the phenomenon for low-eccentricity planetesimals. In fact, it is interesting to note that a 1 Earth mass protoplanet provides resonant torques of sufficient magnitude to halt the orbital decay of planetesimals $-3 \mathrm{~km}$ or larger in radius even in distant resonances such as the $3: 2$. Because resonances closer to the protoplanet provide larger resonant torques, a planetesimal that is too small to get captured in distant (small $j$ ) resonances may eventually be captured in a resonance closer in. 
Given the high likelihood of resonance capture, the stability of the resonance traps with respect to mutual planetesimal interactions is an important consideration for planet formation theories. In the previous section, we provided a quantitative measure of this stability. A detailed discussion of the implications is beyond the scope of the present work (see Torbett et al. 1982, Weidenschilling and Davis 1985 for qualitative discussions on this point), but as an illustration, let us consider the specific case of a $1 M_{\oplus}$ protoplanet at $5 \mathrm{AU}$ and a swarm of 10-km-radius planetesimals. From Eq. (30) we have $(\delta v)_{\mathrm{res}} \approx j^{1 / 4} 6.5 \mathrm{~m} / \mathrm{sec}$. Now, in the planetesimal swarm, random velocities of encounter are on the order of the escape velocity from the surface, which has a similar magnitude, $\sim 10 \mathrm{~m} / \mathrm{sec}$. Furthermore, the relative velocity of encounter between a resonant and a nonresonant planetesimal would be even larger, $\sim e_{\mathrm{eq}} \cdot v_{\mathrm{kep}} \approx(j+1)^{-1 / 2}$. $1 \mathrm{~km} / \mathrm{sec}$, easily exceeding the strength of the resonance. Thus, it is apparent that a planetesimal trapped in a resonance is quite vulnerable to being knocked out of the resonant orbit. However, there are a couple of points to keep in mind: (i) unless it is fragmented, a planetesimal knocked out of one resonance has a high probability of being recaptured in the same or neighboring resonance; (ii) if fragmentation does occur, the bigger fragments will tend to be imparted smaller $\delta v$ 's and so will tend to stay in resonance. We conclude that mutual planetesimal interactions make the resonance barriers permeable, but the resonances may be effective in slowing down the orbital decay.

The above considerations apply to those planetesimals that do get trapped in resonances. Planetesimals that are sufficiently small, $s<s_{0}$, that their rate of orbital decay exceeds the adiabatic threshold for all $j+1: j$ exterior resonances with $j>j_{c}$ (Eq. (28)) will be delivered to the feeding zone of the protoplanet without being slowed down by the resonance traps. By combining Eqs. (5), (20) (with $C_{\mathrm{ad}}=3.3$ ), and (28), we find the critical size, $s_{0}$ to be

$$
\begin{array}{r}
s_{0} \approx 7 \times 10^{3} \mathrm{~cm}\left(\frac{a_{\mathrm{p}}}{5 \mathrm{AU}}\right)\left(\frac{M_{\mathrm{p}}}{M_{\oplus}}\right)^{-3 / 7}\left(\frac{\eta}{0.005}\right)\left(\frac{C_{\mathrm{d}}}{0.5}\right) \\
\left(\frac{\rho_{\mathrm{gas}}}{10^{-10} \mathrm{~g} \mathrm{~cm}^{-3}}\right)\left(\frac{2 \mathrm{~g} \mathrm{~cm}^{-3}}{\rho}\right) .
\end{array}
$$

It is appropriate to recall here that the gas drag law we have adopted (Eqs. (4) and (5)) holds with $C_{\mathrm{d}} \approx$ constant $\approx 0.5$ for high Reynolds numbers. From Eq. (3), we see that this assumption may be in question for planetesimals smaller than the $s_{0}$ determined above. While the form of the drag force is expected to hold for Re as small as $O(10)$, the numerical value of $C_{\mathrm{d}}$ increases steeply with decreasing Re (Landau and Lifshitz 1987). Therefore, we have indicated explicitly in Eq. (31) the dependence of $s_{0}$ on $C_{\mathrm{d}}$. In any case, it may be concluded that the critical size $s_{0}$ is on the order of $100 \mathrm{~m}$ for a $1 M_{\oplus}$ protoplanet.

In summary, we have discussed the trapping of planetesimals in orbital resonances with a protoplanetary embryo in the Solar Nebula. Several idealizations were necessary for elucidating this fundamental dynamical process. Our model included only a single, isolated planetary embryo, assumed to be in a circular orbit, and treated the planetesimals as "test particles"; a constant and uniform nebular gas density was assumed; the response of the gas to the gravity of the protoplanet and planetesimal was neglected. Within this idealized model, we have established the conditions for resonance capture, the stability of the resonances against mutual planetesimal perturbations, and the implications for the delivery of solid bodies to the planetary embryo. However, the idealizations are sufficiently severe that much further work is necessary before the influence of the resonance trapping phenomenon on the late stages of growth of planetary embryos in the Solar Nebula can be fully evaluated.

\section{ACKNOWLEDGMENTS}

I thank the organizers of the Planet Formation program at the Institute for Theoretical Physics (Santa Barbara, CA) for their hospitality during the early stages of this work. I thank David Black, Jack Lissauer, and Tom Stepinski for comments on the first draft of the paper. This research was supported in part by the National Science Foundation under Grant PHY89-04035 and was done while the author was a Staff Scientist at the Lunar and Planetary Institute which is operated by the Universities Space Research Association under contract NASW-4574 with the National Aeronautics and Space Administration. This paper is Lunar and Planetary Institute Contribution 813.

\section{REFERENCES}

Adachi, 1., C. Hayashi, and K. Nakazawa 1976, Prog. Theor. Phys. 56, $1756-1771$.

Borderies. N., AND P. GoldDreICh 1984. A simple derivation of capture probabilities for the $j+1: j$ and $j+2: j$ orbit-orbit resonance problems. Cel. Mech. 32, 127-136.

Brouwer, D., And G. M. Clemence 1961. Methods of Celestial Mechanics. Academic Press, New York.

ChIRIKov, B. V. 1979. A universal instability of many dimensional oscillator systems. Phys. Rep. 52(5), 263-379.

Dermott, S. F., R. Mallhotra, and C. D. Murray 1988. Dynamics of the uranian and saturnian satellite systems: A chaotic route to melting Miranda. licarus 76, 295-334.

HENRARD, J., AND A. LEmaitre 1983. A second fundamental model for resonance. Cel. Mech. 30, 197-218.

Kary, D. M., J. J. Lissauer, and Y. Greenzweig 1993. Nebular Gas Drag and Planetary Accretion. Icartus 106, 288-307.

Landau, L. D., And E. M. Lifshitz 1987. Fluid Mechanics. Pergamon, New York.

Malhotra, R. 1988. Ph.D. dissertation, Cornell University.

Mal.hotra, R. 1993. A mapping method for the few-body problem with dissipation. Celestial Mechanics \& Dynamical Astronomy. in press. 
Malhotra, R., and S. F. Dermott 1990. The role of secondary resonances in the orbital history of Miranda. Icarus 85, 444-480.

PeAle, S. J. 1986. Orbital resonances, unusual configurations and exotic rotation states among the planetary satellites. In Satellites, (J. A Burns and M. S. Matthews, Eds.). Univ. of Arizona Press, Tucson.

Press, W. H., B. P. Flannery, S. A. Teukolsky, and W. T. VetterLING 1986. Numerical Recipes. Cambridge Univ. Press.

Torbett, M., R. Greenberg, And R. Smoluchowsk1 1982. Orbital resonances and planetary formation sites. Icarus 49, 313-326.
Weidenschilling, S. J., ANd Davis, D. R. 1985. Icurus 62, 1629

WetheriLl, G. W. 1989. The formation of the Solar System: Consensus, alternatives, and missing factors. In The Formation and Evolution of Planetary Systems (H. A. Weaver and L. Danly, Eds.). Cambridge University Press.

WisDom, J. 1980. The resonance overlap criterion and the onset of chaotic behavior in the restricted three-body problem. Astron. J. 85, 1122-1133. 
